\title{
Coupled bending-bending-torsion vibration of a pre-twisted beam with aerofoil cross-section by the finite element method
}

\author{
Bulent Yardimoglu ${ }^{1}$ and Daniel J. Inman \\ Center for Intelligent Materials and Structures, Virginia Tech, Virginia, MC 0261, Blacksburg, VA 24061, USA
}

\begin{abstract}
The present study deals with a finite element model for coupled bending-bending-torsion vibration analysis of a pretwisted Timoshenko beam with varying aerofoil cross-section. The element derived in this paper has two nodes, with seven degrees of freedom at each node. The nodal variables are transverse displacements, cross-section rotations and the shear angles in two planes and torsional displacement. The advantage of the present element is the exclusion of unnecessary derivatives of fundamental nodal variables, which were included to obtain invertable square matrix by other researchers, by choosing proper displacement functions and using relationship between cross-sectional rotation and the shear deformation. Element stiffness and mass matrices are developed from strain and kinetic energy expressions by assigning proper order polynomial expressions for cross-section properties and considering higher order coupling coefficients. The correctness of the present model is confirmed by the experimental results available in the literature. Comparison of the proposed model results with those in the literature indicates that a faster convergence is obtained. The results presented also provide some insights in the formulation by clearly indicating that higher order coupling terms have considerable influence on the natural frequencies.
\end{abstract}

\section{Introduction}

Coupled bending-bending-torsion vibrations in beams occur when the center of flexure does not coincide with its centroid, as in the pretwisted beam with aerofoil cross-section. Methods of solution of pretwisted beam coupled bending-bending-torsion vibrations problems are broadly classified into two categories [12], viz. (a) continuum model approach which consists in solving the differential equations directly by approximate methods like Rayleigh-Ritz [2,7], Galerkin [13], collocation [11], transformation [10], Reissner variational method [16,17], and (b) discrete model approach which employs finite difference [4,5], polynomial frequency equation method [12], lumped parameters [6] and finite element $[1,8,9,14,15]$. The finite element method is the most versatile method for accurate prediction when the system is modeled prop-

\footnotetext{
${ }^{1}$ On leave from Department of Mechanical Engineering, Izmir Institute of Technology, Izmir, Turkey.
}

erly. Even with intensive development of finite element models to date, considerable differences exist between numerical and experimental results. The governing differential equations for coupled bending-bendingtorsion vibration have been derived and studied extensively for various idealized cases by Carnegie et al. [25,13]. Montoya [10] derived governing equations of motion for the same problem including shear center and higher order effects and solved these by the RungeKutta numerical integration method. In his treatment, an additional term appears in the bending moment and torsional moment equations due to torsion and bending, respectively. Some Timoshenko beam finite elements have been proposed in the literature $[1,8,9]$. Karadag [8,9] employed an element having eighteen degrees of freedom. Nodal variables are bending and shear displacements with their first derivatives in two planes and torsional displacement. The shape functions are classical third degree polynomials. Abbas and Kamal [1] used an element with twenty-four degrees of freedom, comprising values of deflections and bend- 
ing slopes in two planes, axial displacement, torsional displacement, and their first derivatives at the nodes, assuming cubic polynomial expansions.

The purpose of this paper is to introduce a finite element model for coupled bending-bending-torsion vibration of a pretwisted Timoshenko beam model of a varying aerofoil cross section blade. The proposed model is based on; the Timoshenko beam finite element developed for straight untwisted beams, derived and proven by Thomas et al. [18], coupled bendingbending-torsion moment equations derived by Montoya [10] and also partly on the blade energy expressions allowing for rotary inertia and shear deflection derived by Carnegie [3,5]. The nodal variables in the present element are transverse displacements, cross section rotations and shear angles in two planes and torsional displacement. The transverse displacements are interpolated as cubic polynomial functions. Shear angles and torsional displacements are assumed to vary linearly. Cross section rotations are related with other parameters by Timoshenko shear angle expression [19, p. 745, Eq. (3)]. The coupled bending and torsional vibration problem in a pretwisted blade treated experimentally and solved numerically by Montoya [10] is selected to demonstrate the accuracy and convergence of the present model, and to clarify the influence of higher order coupling coefficients. Cross-section properties of the blade given by Montoya [10] at nine discrete cross-sections along the blade are expressed with proper order polynomial functions determined by curve fitting. Mass and stiffness matrices are evaluated by numerical integration.

\section{Moment equations}

When a pretwisted beam of asymmetrical aerofoil cross-section, as shown in Fig. 1, is subjected to vibration movement, the motion consists of coupled bending-bending-torsion vibration and causes bending and torsional moments which are determined by the following expressions [10]:

$$
\begin{aligned}
& M_{x}=-E I_{G x x} \theta_{x}^{\prime}-E I_{G x y} \theta_{y}^{\prime}-E J_{x} \alpha \theta_{z}^{\prime} \\
& M_{y}=E I_{G x y} \theta_{x}^{\prime}+E I_{G x y} \theta_{y}^{\prime}+E J_{y} \alpha \theta_{z}^{\prime} \\
& M_{z}=\left(G I_{T}+E J \alpha^{2}\right) \theta_{z}^{\prime}+E \alpha\left(J_{x} \theta_{x}^{\prime}+J_{y} \theta_{y}^{\prime}\right)
\end{aligned}
$$

A list of notation is given in Appendix A. The area moments of inertia of the cross-section are related to the principal area moments of inertia by

$$
\begin{aligned}
& I_{G x x}(z)=I_{G \xi \xi}(z) \cos ^{2} \phi(z)+I_{G \eta \eta} \sin ^{2} \phi(z)(4) \\
& I_{G y y}(z)=I_{G \xi \xi}(z) \sin ^{2} \phi(z)+I_{G \eta \eta} \cos ^{2} \phi(z)(5) \\
& I_{G x y}(z)=0.5\left(I_{G \eta \eta}(z)-I_{G \xi \xi}(z)\right) \sin 2 \phi(z)(6) \\
& \text { where } \phi(z)=\phi_{0}+\alpha(z) z
\end{aligned}
$$

Coefficients of coupling are given as follows [10]:

$$
\begin{aligned}
& J_{x}(z)=J_{\xi}(z) \cos \phi(z)+J_{\eta} \sin \phi(z) \\
& J_{y}(z)=-J_{\xi}(z) \sin \phi(z)+J_{\eta} \cos \phi(z) \\
& J=J_{T}-\left(I_{T P}^{2} / A\right)
\end{aligned}
$$

where

$$
\begin{aligned}
& J_{\xi}=r_{\eta} I_{T P}-J_{T \xi} \\
& J_{\eta}=r_{\xi} I_{T P}-J_{T \eta}
\end{aligned}
$$

in which $J_{T \xi}, J_{T \eta}$ and $J_{T}$ are given in Appendix B.

\section{Strain energy}

By using the approach given by reference [5, p. 277], considering the moments produce angular displacements and shear forces produce shear deflections, the strain energy of an element is found as follows:

$$
\begin{aligned}
U= & 0.5 \int_{0}^{L} E\left(I_{G x x} \theta_{{ }^{\prime}}^{2}+2 I_{G x y} \theta^{\prime}{ }_{x} \theta^{\prime}{ }_{y}\right. \\
& \left.+I_{G y y}{\theta^{\prime}}_{y}^{2}\right) d z \\
& +\int_{0}^{L} E \alpha\left(J_{x} \theta^{\prime}{ }_{x}+J_{y} \theta^{\prime}{ }_{y}\right) \theta^{\prime}{ }_{z} d z \\
& +0.5 \int_{0}^{L}\left(G I_{T}+E J \alpha^{2}\right){\theta^{\prime}}_{z}^{2} d z \\
& +0.5 \int_{0}^{L} k A G\left(\psi_{x}^{2}+\psi_{y}^{2}\right) d z
\end{aligned}
$$

where $\psi_{x}=v^{\prime}-\theta_{x}$ and $\psi_{y}=u^{\prime}-\theta_{y}$

The symbol " / " used in Eqs (13) and (14) represents differentiation with respect to $z$. 


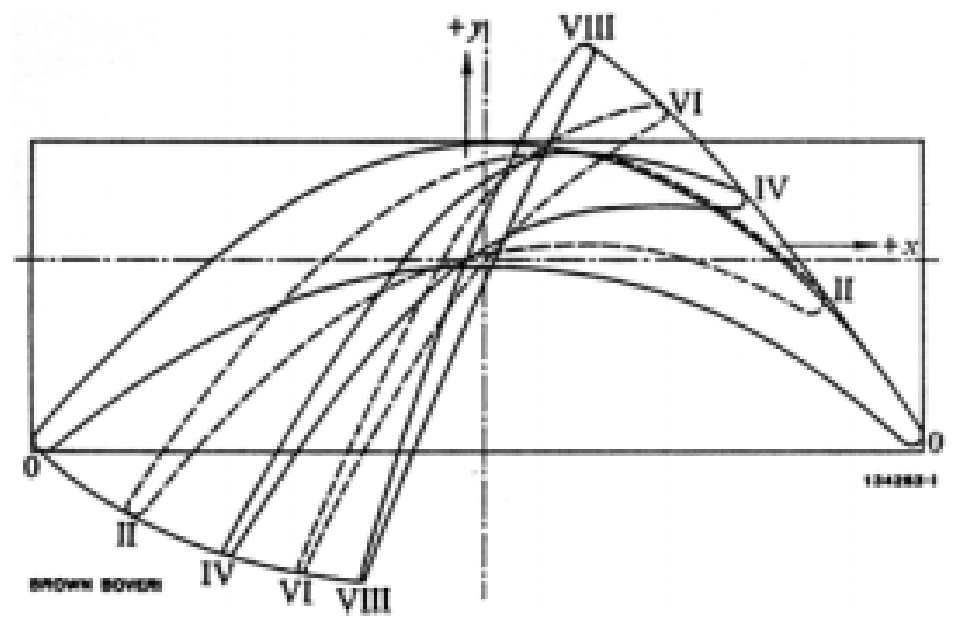

Fig. 1. View with cross-sections looking axially along a pretwisted turbine blade from tip toward root cross-section (see Fig. 2 for co-ordinate systems).

\section{Kinetic energy}

Kinetic energy of an element is obtained considering reference $[5$, p. 277] and reference $[3$, p. 106] as follows:

$$
\begin{aligned}
T= & 0.5 \int_{0}^{L} \rho A\left(v_{G x}^{2}+v_{G y}^{2}\right) d z \\
& +0.5 \int_{0}^{L} \rho\left(I_{G x x} \omega_{x}^{2}+2 I_{G x y} \omega_{x} \omega_{y}\right. \\
& \left.+I_{G y y} \omega_{y}^{2}\right) d z+0.5 \int_{0}^{L} \rho I_{G P} \omega_{z}^{2} d z
\end{aligned}
$$

where

$$
\begin{aligned}
& v_{G x}=\dot{u}-r_{y} \dot{\theta}_{z} \\
& v_{G y}=\dot{v}+r_{x} \dot{\theta}_{z} \\
& \omega_{x}=\dot{\theta}_{x}+r_{x} \dot{\theta}_{z}^{\prime}+r_{x}^{\prime} \dot{\theta}_{z} \\
& \omega_{y}=\dot{\theta}_{y}-r_{y} \dot{\theta}_{z}^{\prime}+r_{x}^{\prime} \dot{\theta}_{z} \\
& \omega_{z}=\dot{\theta}_{z}
\end{aligned}
$$

in which

$$
\begin{aligned}
r_{x}(z)= & r_{\xi}(z) \cos \phi(z)-r_{\eta} \sin \phi(z) \\
r_{y}(z)= & r_{\xi}(z) \sin \phi(z)+r_{\eta} \cos \phi(z) \\
r_{x}^{\prime}(z)= & {\left[r_{\xi}^{\prime}(z)-r_{\eta}(z) \alpha(z)\right] \cos \phi(z) } \\
& -\left[r_{\eta}^{\prime}(z)+r_{\xi}(z) \alpha(z)\right] \sin \phi(z) \\
r_{y}^{\prime}(z)= & {\left[r_{\xi}^{\prime}(z)-r_{\eta}(z) \alpha(z)\right] \sin \phi(z) } \\
& +\left[r_{\eta}^{\prime}(z)+r_{\xi}(z) \alpha(z)\right] \cos \phi(z)
\end{aligned}
$$

The overdot used in Eqs (16) to (20) is a compact notation for differentiation with respect to time.

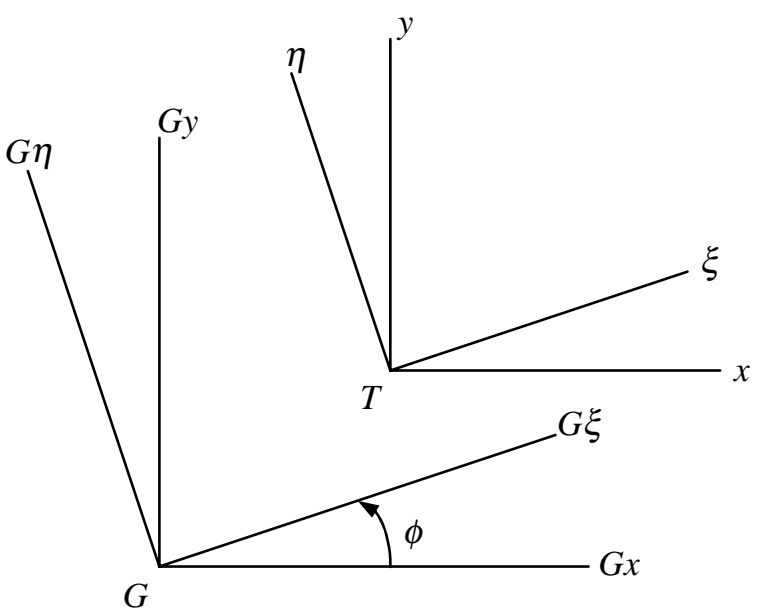

Fig. 2. Co-ordinate systems of a pretwisted beam cross-section. G: centroid; T: shear center.

\section{Finite element formulation}

The element derived in this section, extending model in reference [18], has two nodes, with seven degrees of freedom at each node. The nodal variables are transverse displacements, cross section rotations and shear angles in two planes and torsional displacement. The transverse displacements are interpolated as cubic polynomial functions. Shear angles and torsional displacements are assumed to vary linearly.

Cross section rotations are given by Timoshenko [19, p. 745, (3)] as follows:

$$
\theta_{x}=v^{\prime}-\psi_{x} \text { and } \theta_{y}=u^{\prime}-\psi_{y}
$$

Hence, by using Eq. (25), nodal displacement vector can be written in the form 


$$
\left\{q_{n}\right\}=[Z]\{a\}
$$

where

$$
\begin{aligned}
& \left\{q_{n}\right\}=\left\{\begin{array}{lllllll}
u & v & \theta_{x} & \theta_{y} & \psi_{x} & \psi_{y} & \theta_{z}
\end{array}\right\}^{T} \\
& \{a\}=\left\{\begin{array}{llllllllll}
a_{0} & a_{1} & a_{2} & a_{3} & b_{0} & b_{1} & b_{2} & b_{3} & c_{0} & c_{1}
\end{array}\right. \\
& \left.\begin{array}{llll}
d_{0} & d_{1} & e_{0} & e_{1}
\end{array}\right\}^{T} \\
& \{Z\}=
\end{aligned}
$$

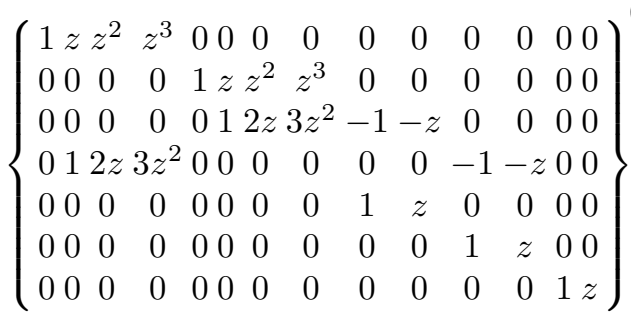

Therefore, polynomial vectors for each nodal variable can be expressed in closed form by separating the each rows in Eq. (29) as

$$
\begin{aligned}
& {\left[P_{d r}\right]=\left[Z_{r, c}\right] ; r=1,2, \ldots, 7, c=1,2, \ldots, 14} \\
& d r \equiv u, v, \theta_{x}, \theta_{y}, \psi_{x}, \psi_{y}, \theta_{z}
\end{aligned}
$$

The element displacement vector is related to the polynomial coefficient vector by

$$
\left\{q_{e}\right\}=[C]\{a\}
$$

where

$$
\begin{aligned}
& \left\{q_{e}\right\}=\left\{\begin{array}{l}
\left\{q_{n 1}\right\} \\
\left\{q_{n 2}\right\}
\end{array}\right\} \\
& {[C]=\left[\begin{array}{l}
{[Z]_{z=0}} \\
{[Z]_{z=L}}
\end{array}\right]}
\end{aligned}
$$

Now, substituting Eqs (26) and (31) into (13) gives

$$
\begin{aligned}
& U=0.5\left\{q_{e}\right\}^{T}\left[K_{e}\right]\left\{q_{e}\right\} \\
& \text { where }\left[K_{e}\right]=[C]^{-T}\left[k_{e}\right][C]^{-1}
\end{aligned}
$$

in which

$$
\begin{aligned}
{\left[k_{e}\right]=} & E I_{G x x}\left[P_{\theta_{x}}^{\prime}\right]^{T}\left[P_{\theta_{x}}^{\prime}\right]+E I_{G y y}\left[P_{\theta_{y}}^{\prime}\right]^{T}\left[P_{\theta_{y}}^{\prime}\right] \\
& +E I_{G x y}\left(\left[P_{\theta_{x}}^{\prime}\right]^{T}\left[P_{\theta_{y}}^{\prime}\right]+\left[P_{\theta_{y}}^{\prime}\right]^{T}\left[P_{\theta_{x}}^{\prime}\right]\right) \\
& +E \alpha J_{x}\left(\left[P_{\theta_{x}}^{\prime}\right]^{T}\left[P_{\theta_{z}}^{\prime}\right]+\left[P_{\theta_{z}}^{\prime}\right]^{T}\left[P_{\theta_{x}}^{\prime}\right]\right) \\
& +E \alpha J_{y}\left(\left[P_{\theta_{y}}^{\prime}\right]^{T}\left[P_{\theta_{z}}^{\prime}\right]+\left[P_{\theta_{z}}^{\prime}\right]^{T}\left[P_{\theta_{y}}^{\prime}\right]\right) \\
& +\left(G I_{T}+E J \alpha^{2}\right)\left[P_{\theta_{z}}^{\prime}\right]^{T}\left[P_{\theta_{z}}^{\prime}\right] \\
& +k A G\left(\left[P_{\psi x}\right]^{T}\left[P_{\psi x}\right]+\left[P_{\psi y}\right]^{T}\left[P_{\psi y}\right]\right)
\end{aligned}
$$

Also, substituting Eqs (26) and (31) into (15) gives

$$
T=0.5\left\{\dot{q}_{e}\right\}^{T}\left[M_{e}\right]\left\{\dot{q}_{e}\right\}
$$

$$
\text { where }\left[M_{e}\right]=[C]^{-T}\left[m_{e}\right][C]^{-1}
$$

in which

$$
\begin{aligned}
{\left[m_{e}\right]=} & \rho\left(A\left[m_{A}\right]+I_{G x x}\left[m_{G x x}\right]\right. \\
& +I_{G x y}\left[m_{G x y}\right]+I_{G y y}\left[m_{G y y}\right] \\
& \left.+I_{G P}\left[m_{G P}\right]\right) \\
{\left[m_{A}\right]=} & {\left[\dot{P}_{u}\right]^{T}\left[\dot{P}_{u}\right]+\left[\dot{P}_{v}\right]^{T}\left[\dot{P}_{v}\right] } \\
& +\left(r_{x}^{2}+r_{y}^{2}\right)\left[\dot{P}_{\theta_{z}}\right]^{T}\left[\dot{P}_{\theta_{z}}\right] \\
& +r_{x}\left(\dot{P}_{v}\right]^{T}\left[\dot{P}_{\theta_{z}}\right]+\left[\dot{P}_{\theta_{z}}\right]^{T}\left[\dot{P}_{v}\right] \\
& -r_{y}\left(\left[\dot{P}_{u}\right]^{T}\left[\dot{P}_{\theta_{z}}\right]+\left[\dot{P}_{\theta_{z}}\right]^{T}\left[\dot{P}_{u}\right]\right)
\end{aligned}
$$

$$
\begin{aligned}
{\left[m_{G x x}\right]=} & {\left[\dot{P}_{\theta_{x}}\right]^{T}\left[\dot{P}_{\theta_{x}}\right] } \\
& +r_{x}^{2}\left[\dot{P}_{\theta_{z}}^{\prime}\right]^{T}\left[\dot{P}_{\theta_{z}}^{\prime}\right]+r_{x}^{\prime 2}\left[\dot{P}_{\theta_{z}}\right]^{T}\left[\dot{P}_{\theta_{z}}\right] \\
& +r_{x}\left(\left[\dot{P}_{\theta_{x}}\right]^{T}\left[\dot{P}_{\theta_{z}}^{\prime}\right]+\left[\dot{P}_{\theta_{z}}^{\prime}\right]^{T}\left[\dot{P}_{\theta_{x}}\right]\right) \\
& +r_{x}^{\prime}\left(\left[\dot{P}_{\theta_{x}}\right]^{T}\left[\dot{P}_{\theta_{z}}\right]+\left[\dot{P}_{\theta_{z}}\right]^{T}\left[\dot{P}_{\theta_{x}}\right]\right) \\
& +r_{x} r_{x}^{\prime}\left(\left[\dot{P}_{\theta_{z}}^{\prime}\right]^{T}\left[\dot{P}_{\theta_{z}}\right]+\left[\dot{P}_{\theta_{z}}\right]^{T}\left[\dot{P}_{\theta_{z}}^{\prime}\right]\right)
\end{aligned}
$$

$$
\left[m_{G x y}\right]=\left(\left[\dot{P}_{\theta_{x}}\right]^{T}\left[\dot{P}_{\theta_{y}}\right]+\left[\dot{P}_{\theta_{y}}\right]^{T}\left[\dot{P}_{\theta_{x}}\right]\right)
$$$$
+r_{x}\left(\left[\dot{P}_{\theta_{z}}^{\prime}\right]^{T}\left[\dot{P}_{\theta_{y}}\right]+\left[\dot{P}_{\theta_{y}}\right]^{T}\left[\dot{P}_{\theta_{z}}^{\prime}\right]\right)
$$$$
+r^{\prime}{ }_{x}\left(\left[\dot{P}_{\theta_{z}}\right]^{T}\left[\dot{P}_{\theta_{y}}\right]+\left[\dot{P}_{\theta_{y}}\right]^{T}\left[\dot{P}_{\theta_{z}}\right]\right)
$$$$
-r_{y}\left(\left[\dot{P}_{\theta_{x}}\right]^{T}\left[\dot{P}_{\theta_{z}}^{\prime}\right]+\left[\dot{P}_{\theta_{z}}^{\prime}\right]^{T}\left[\dot{P}_{\theta_{x}}\right]\right)
$$$$
-r_{y}^{\prime}\left(\left[\dot{P}_{\theta_{x}}\right]^{T}\left[\dot{P}_{\theta_{z}}\right]+\left[\dot{P}_{\theta_{z}}\right]^{T}\left[\dot{P}_{\theta_{z}}\right]\right.
$$$$
-2 r_{x} r_{y}\left[\dot{P}_{\theta_{z}}^{\prime}\right]^{T}\left[\dot{P}_{\theta_{z}}^{\prime}\right]-2 r_{x}^{\prime} r_{y}^{\prime}\left[\dot{P}_{\theta_{z}}\right]^{T}\left[\dot{P}_{\theta_{z}}\right]
$$$$
-\left(r_{x} r^{\prime}{ }_{y}+r_{x}^{\prime} r_{y}\right)\left(\left[\dot{P}_{\theta_{z}}^{\prime}\right]^{T}\left[\dot{P}_{\theta_{z}}\right]\right.
$$$$
\left.+\left[\dot{P}_{\theta_{z}}\right]^{T}\left[\dot{P}_{\theta_{z}}^{\prime}\right]\right)
$$

$$
\begin{aligned}
{\left[m_{G y y}\right]=} & {\left[\dot{P}_{\theta_{y}}\right]^{T}\left[\dot{P}_{\theta_{y}}\right] } \\
& +r_{y}^{2}\left[\dot{P}_{\theta_{z}}^{\prime}\right]^{T}\left[\dot{P}_{\theta_{z}}^{\prime}\right]+r_{y}^{\prime 2}\left[\dot{P}_{\theta_{z}}\right]^{T}\left[\dot{P}_{\theta_{z}}\right] \\
& -r_{y}\left(\left[\dot{P}_{\theta_{y}}\right]^{T}\left[\dot{P}_{\theta_{z}}^{\prime}\right]+\left[\dot{P}_{\theta_{z}}^{\prime}\right]^{T}\left[\dot{P}_{\theta_{y}}\right]\right) \\
& -r_{y}^{\prime}\left(\left[\dot{P}_{\theta_{y}}\right]^{T}\left[\dot{P}_{\theta_{z}}\right]+\left[\dot{P}_{\theta_{z}}\right]^{T}\left[\dot{P}_{\theta_{y}}\right]\right) \\
& +r_{y} r_{y}^{\prime}\left(\left[\dot{P}_{\theta_{z}}^{\prime}\right]^{T}\left[\dot{P}_{\theta_{z}}\right]+\left[\dot{P}_{\theta_{z}}\right]^{T}\left[\dot{P}_{\theta_{z}}^{\prime}\right]\right)
\end{aligned}
$$

$$
\left[m_{G P}\right]=\left[\dot{P}_{\theta_{z}}\right]^{T}\left[\dot{P}_{\theta_{z}}\right]
$$


Table 1

Aerofoil cross-section properties of a $250 \mathrm{MW}$ turbine blade $L_{\text {blade }}=71.65 \mathrm{~cm}, E=201.105 \mathrm{GPa}, G=78.48 \mathrm{GPa}, \rho=7740 \mathrm{~kg} / \mathrm{m}^{3}[10]$

\begin{tabular}{|c|c|c|c|c|c|c|c|c|c|}
\hline Section number & 0 & 1 & 2 & 3 & 4 & 5 & 6 & 7 & 8 \\
\hline $\mathrm{A}\left(\mathrm{cm}^{2}\right)$ & 28.37 & 22.0 & 16.47 & 11.84 & 8.689 & 7.018 & 5.884 & 5.018 & 4.258 \\
\hline$I_{G \xi \xi}\left(\mathrm{cm}^{4}\right)$ & 48.27 & 31.47 & 18.12 & 9.468 & 4.626 & 2.175 & 0.876 & 0.3054 & 0.0934 \\
\hline$I_{G \eta \eta}\left(\mathrm{cm}^{4}\right)$ & 470.8 & 299.3 & 189.0 & 113.2 & 74.77 & 55.45 & 44.88 & 37.86 & 34.15 \\
\hline$\phi(\mathrm{rad})$ & 0.0145 & 0.1548 & 0.3243 & 0.4837 & 0.6528 & 0.8046 & 0.9354 & 1.064 & 1.179 \\
\hline$I_{T}\left(\mathrm{~cm}^{4}\right)$ & 30.64 & 17.31 & 8.772 & 4.373 & 2.152 & 1.237 & 0.8033 & 0.4988 & 0.2743 \\
\hline$\xi_{T}(\mathrm{~cm})$ & -0.073 & 0.1033 & 0.373 & 0.4212 & 0.5218 & 0.7091 & 0.7803 & 0.76 & 0.6812 \\
\hline$\eta_{T}(\mathrm{~cm})$ & 1.863 & 1.67 & 1.462 & 1.329 & 1.012 & 0.7728 & 0.5170 & 0.2404 & 0.129 \\
\hline$J_{G}\left(\mathrm{~cm}^{6}\right)$ & 19750 & 10530 & 5697 & 2975 & 1764 & 1145 & 857.3 & 665.6 & 600 \\
\hline$J_{G \xi}\left(\mathrm{cm}^{5}\right)$ & -623 & -368.8 & -199.9 & -102.2 & -52.76 & -28.93 & -15.29 & -7.18 & -2.586 \\
\hline$J_{G \eta}\left(\mathrm{cm}^{5}\right)$ & -163.8 & -227.9 & -253.5 & -187.4 & -137.5 & -89.86 & -69.28 & -46.94 & -36.59 \\
\hline
\end{tabular}

Table 2

Convergence and comparison of coupled bending-bending-torsion vibrations of a $250 \mathrm{MW}$ turbine blade with aerofoil cross-section; blade properties are given in Table 1

\begin{tabular}{|c|c|c|c|c|c|c|c|}
\hline \multirow{2}{*}{$\begin{array}{l}\text { Number of element } \\
\text { (degrees of freedom) }\end{array}$} & \multicolumn{7}{|c|}{ Natural Frequency $(\mathrm{Hz})$} \\
\hline & First bending & Second bending & First torsion & Third bending & Fourth bending & Second torsion & Third torsion \\
\hline $2(14)$ & 83.01 & 194.45 & 386.17 & 443.33 & 732.57 & 794.56 & 1530.01 \\
\hline $4(28)$ & 80.50 & 186.76 & 368.36 & 413.61 & 555.34 & 762.07 & 951.30 \\
\hline $6(42)$ & 79.91 & 184.30 & 362.96 & 405.15 & 539.49 & 736.32 & 935.39 \\
\hline $8(56)$ & 79.70 & 183.27 & 360.90 & 401.30 & 531.90 & 727.87 & 911.45 \\
\hline $10(70)$ & 79.59 & 182.82 & 359.91 & 399.95 & 528.60 & 723.89 & 894.56 \\
\hline $12(84)$ & 79.52 & 182.54 & 359.36 & 399.29 & 527.04 & 721.60 & 886.92 \\
\hline $14(98)$ & 79.48 & 182.34 & 359.03 & 398.83 & 526.06 & 720.16 & 882.57 \\
\hline $16(112)$ & 79.45 & 182.20 & 358.81 & 398.52 & 525.39 & 719.20 & 879.57 \\
\hline Experimental [10] & 83.4 & 184.8 & 344.3 & 399.7 & 500 & 655 & 840 \\
\hline Calculated [10] & 80.3 & 185.5 & 356.3 & 410.3 & 535.7 & 724.3 & 847.1 \\
\hline $7(63)[8,9]$ & 79.93 & 184.39 & 362.13 & 401.2 & 535.67 & 727.66 & 916.16 \\
\hline $5(60)[1]$ & 79.45 & 179.08 & 360.75 & 399.55 & 511.40 & 733.61 & 883.61 \\
\hline
\end{tabular}

Table 3

The effects of coupling coefficients on the natural frequencies (Number of element $=8$ )

\begin{tabular}{lcccccc}
\hline Case & \multicolumn{5}{c}{ Natural frequency (Hz) } \\
\cline { 2 - 7 } & First bending & Second bending & First torsion & Third bending & Fourth bending & Second torsion Third torsion \\
\hline$J_{x}=0, J_{y}=0, J=0$ & 92.56 & 199.94 & 302.25 & 434.62 & 602.52 & 614.58 \\
$J_{x} \neq 0, J_{y} \neq 0, J \neq 0$ & 79.70 & 183.27 & 360.90 & 401.30 & 531.90 & 727.87 \\
Experimental [10] & 83.40 & 184.80 & 344.30 & 399.70 & 500.00 & 655.00 \\
Calculated by ignoring [10] & 93.58 & 206.90 & 303.50 & 441.22 & 621.70 & 626.70 \\
\hline
\end{tabular}

\section{Dynamic equilibrium equation}

Element stiffness and mass matrices derived in the previous section are assembled in the usual way with clamped-free boundary conditions to form the global matrices. In order to obtain the natural frequencies, dynamic equilibrium equation is reduced to eigenvalue problem given below,

$$
\left([K]-\Omega^{2}[M]\right)\{q\}=0
$$

The eigenvalue problem is then solved numerically.

\section{Analysis and discussion}

As an application example, the $250 \mathrm{MW}$ turbine blade with aerofoil cross section, shown in Fig. 1, analyzed experimentally by Montoya [10] is considered. Cross-section properties of the blade given in Table 1 at nine discrete cross-sections along the blade are expressed as eighth, seventh and sixth-order polynomial functions determined by curve fitting. The polynomial functions are carefully checked by calculating the coefficients of determination [20, p. 445, eq.(17.10)], also by inspecting the plots of functions and the data. The 
coefficients of determination of eighth-order polynomial functions are equal to unity, i.e. they fully satisfy the data at discrete points. The $r^{2}$ values for $J_{G \eta}$ expressed as seventh-order, $\xi_{T}$ and $\eta_{T}$ expressed as sixthorder are $0.998,0.9963$ and 0.9987 respectively. The coefficients of these functions are given in Table 4 in the Appendix C. Also, some relationships are derived to transfer these data from centroid to shear center considering the co-ordinate systems shown in Fig. 2 and are given in the Appendix B. Convergence study results of present model for the first seven natural frequencies, experimental and calculated results reported by Montoya [10] and other finite element model results [1,8,9] are given in Table 2. Mass and stiffness matrices are calculated by Gauss-Legendre integration in Matlab. Table 2 shows that the present results obtained by employing considerably less degrees of freedom converge rapidly, and are in good agreement with the experimental results. In order to clarify the effects of higher order coupling coefficients on the natural frequencies, analysis is fulfilled ignoring these coefficients. The latter results are given in Table 3 to compare with the experimental and other results. It is clear from Table 3 that these coefficients are quite influential on natural frequencies.

\section{Conclusions}

The finite element model derived in this paper is found to be appropriate for coupled bending-bendingtorsion vibration analysis of varying aerofoil crosssectional pretwisted beam having any form of pretwist. The results obtained by using a quite modest number of degrees of freedom give good accuracy when compared with the experimental and other investigator's results. Also, neglecting the coefficient of coupling leads to erroneous results.

\section{References}

[1] B.A.H. Abbas and K.M. Kamal, Vibration of Turbomachinery blades with root flexibility effect, Bladed Disk Assemblies ASME DE-6 (1987), 31-41.

[2] W. Carnegie, Vibrations of pre-twisted cantilever blading, Proceedings of the Institution of Mechanical Engineers 173(12) (1959), 343-374.

[3] W. Carnegie, Vibrations of pre-twisted cantilever blading allowing for rotary inertia and shear deflection, Journal of Mechanical Engineering Science 6(2) (1964), 105-109.
[4] W. Carnegie and B. Dawson, Vibration characteristics of pretwisted blades of asymmetrical aerofoil cross-section, The Aeronautical Quarterly 22 (1971), 257-273.

[5] W. Carnegie and J. Thomas, The effects of shear deformation and rotary inertia on the lateral frequencies of cantilever beams in bending, Journal of Engineering for Industry 94 (1972), 267-278.

[6] C.C. Fu, Computer analysis of a rotating axial-turbomachinary blade in coupled bending-bending-torsion vibrations, International Journal for Numerical Methods in Engineering $\mathbf{8}$ (1974), 569-588.

[7] J.C. Houbolt and G.W. Brooks, Differential equations of motion for combined flapwise bending chordwise bending and torsion of twisted nonuniform rotor blades, NACA Report 1346, 1958.

[8] V. Karadag, Finite element dynamic analysis of blade shear center effects on practical bladed disc, Journal of Sound and Vibration 94(2) (1984), 183-197.

[9] V. Karadag, Dynamic analysis of practical blades with shear center effect, Journal of Sound and Vibration 94(4) (1984), $471-490$.

[10] J. Montoya, Coupled bending and torsional vibrations in a twisted rotating blade, The Brown Boveri Review 53(3) (1966), 216-230.

[11] J.S. Rao, Coupled bending bending torsion vibrations of cantilever beams, Journal of the Aeronautical Society of India 24(1) (1972), 265-268.

[12] J.S. Rao and S. Banerjee, Coupled bending-torsional vibrations of rotating cantilever blades-method of polynomial frequency equation, Mechanism and Machine Theory 12 (1977), 271-280.

[13] J.S. Rao and W. Carnegie, Solution of the equations of motion of coupled-bending bending torsion vibrations of turbine blades by the method of Ritz-Galerkin, International Journal of Mechanical Sciences 12 (1970), 875-882.

[14] M. Sabuncu, Coupled vibration analysis of blades with angular pretwist of cubic distribution, AIAA Journal 23(9) (1985), 1424-1430.

[15] M. Sabuncu and J.Thomas, Vibration characteristics of pretwisted aerofoil cross-section blade packets under rotating conditions, AIAA Journal 30(1) (1992), 241-250.

[16] K.B. Subrahmanyam, S.V. Kulkarni and J.S.Rao, Coupled bending-torsion vibrations of rotating blades of asymmetric aerofoil cross section with allowance for shear deflection and rotary inertia by use of The Reissner Method, Journal of Sound and Vibration 75(1) (1981), 17-36.

[17] K.B. Subrahmanyam, S.V. Kulkarni and J.S.Rao, Application of the Reissner Method to derive the coupled bending-torsion equations of dynamic motion of rotating pretwisted cantilever blading with allowance for shear deflection, rotary inertia, warping and thermal effects, Journal of Sound and Vibration 84(2) (1982), 223-240.

[18] D.L. Thomas, J.M. Wilson and R.R. Wilson, Timoshenko beam finite elements, Journal of Sound and Vibration 31(3) (1973), 315-330.

[19] S.P. Timoshenko, On the correction for shear of the differential equation for transverse vibration of prismatic bars, Philosophical Magazine 41 (1921), 744-746.

[20] S.C. Chapra and R.P. Canale, Numerical Methods for Engineers: With Programming and Software Applications, Third edition, WCB/Mc-Graw Hill, 1998. 


\section{Appendix A: Notation}

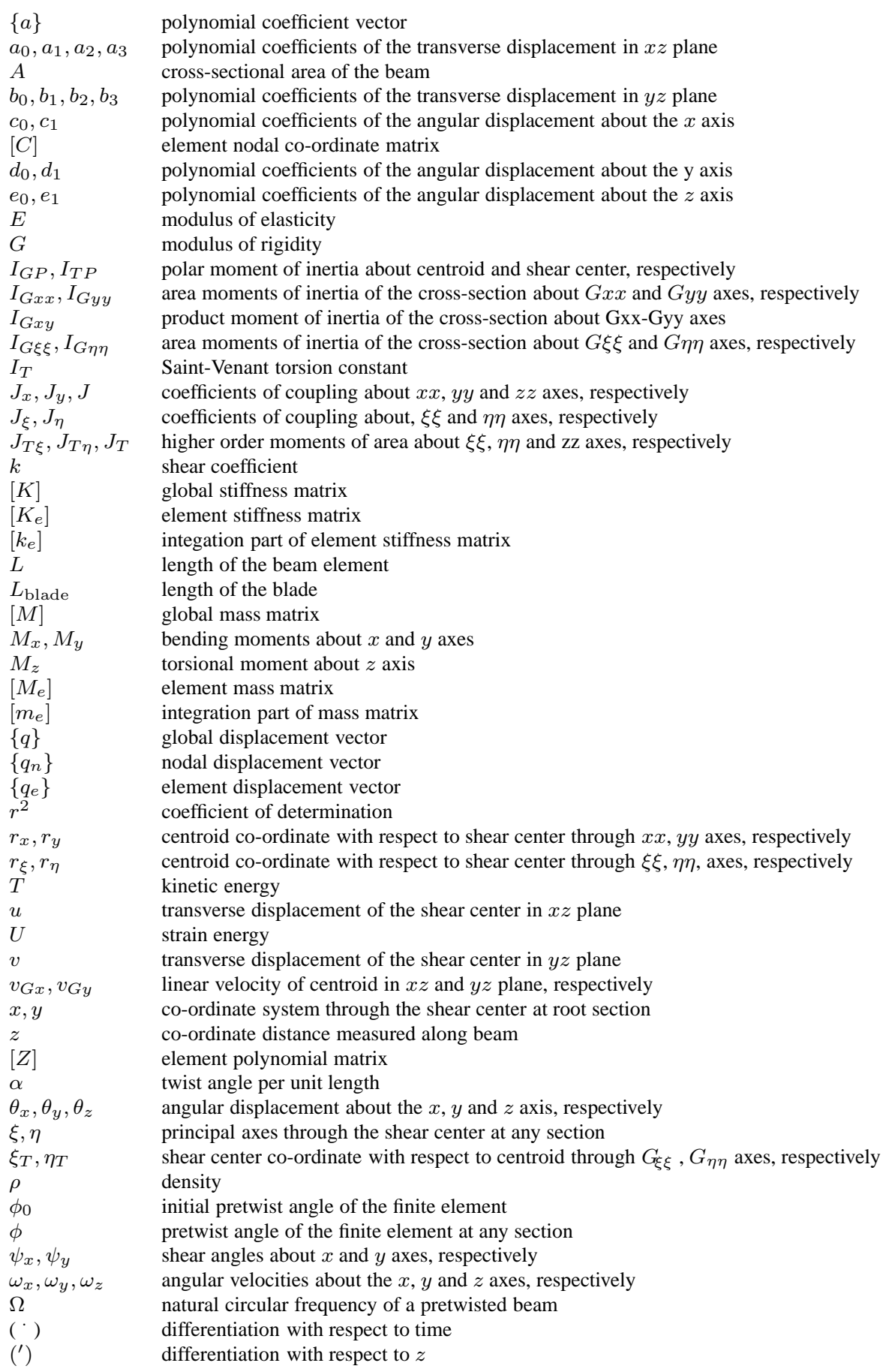




\section{Appendix B}

$$
\begin{aligned}
& J_{T \xi}=\int_{A} \eta\left(\xi^{2}+\eta^{2}\right) d A \\
& J_{T \eta}=\int_{A} \xi\left(\xi^{2}+\eta^{2}\right) d A \\
& J_{T}=\int_{A}\left(\xi^{2}+\eta^{2}\right)^{2} d A \\
& I_{T P}=I_{G P}+\left(r_{\xi}^{2}+r_{\eta}^{2}\right) A \\
& J_{T \xi}=J_{G \xi}+3 r_{\eta} I_{G \xi \xi}+r_{\eta} I_{G \eta \eta}+\left(r_{\eta} r_{\xi}^{2}+r_{\eta}^{3}\right) A \\
& J_{T \eta}=J_{G \eta}+3 r_{\xi} I_{G \eta \eta}+r_{\xi} I_{G \xi \xi}+\left(r_{\xi} r_{\eta}^{2}+r_{\xi}^{3}\right) A \\
& J_{T}=J_{G}+\left(6 r_{\xi}^{2}+2 r_{\eta}^{2}\right) I_{G \eta \eta}+\left(6 r_{\eta}^{2}+2 r_{\xi}^{2}\right) I_{G \xi \xi}+\left(r_{\xi}^{2}+r_{\eta}^{2}\right)^{2} A+4 r_{\eta} J_{G \xi}+4 r_{\xi} J_{G \eta}
\end{aligned}
$$

\section{Appendix C}

Table 4

(a) The polynomial coefficients of cross-section parameters:

$p(x)=p_{n} x^{n}+p_{n-1} x^{x-1}+\ldots+p_{1} x+p_{0}$

\begin{tabular}{rrrrrr}
\hline$p_{i}$ & $A\left(\mathrm{~cm}^{2}\right)$ & $I_{G \xi \xi}\left(\mathrm{cm}^{4}\right)$ & $I_{G \eta \eta}\left(\mathrm{cm}^{4}\right)$ & $\phi(\mathrm{rad})$ & $I_{T}\left(\mathrm{~cm}^{4}\right)$ \\
\hline$p_{0}$ & 28.37 & 48.27 & 470.8 & 0.0145 & 30.64 \\
$p_{1}$ & -0.712824377762 & -1.86692094507 & -30.8137507062 & $-2.36675638856 \mathrm{e}-3$ & -1.45064878875 \\
$p_{2}$ & -0.0103811078578 & -0.0232742600289 & 2.43413451178 & $4.3014135439 \mathrm{e}-3$ & -0.0518565494345 \\
$p_{3}$ & $2.0900213884 \mathrm{e}-3$ & $3.41043743084 \mathrm{e}-3$ & -0.187860645211 & $-3.80517229206 \mathrm{e}-4$ & $8.13166301003 \mathrm{e}-3$ \\
$p_{4}$ & $-1.38886520452 \mathrm{e}-4$ & $-1.25401193994 \mathrm{e}-4$ & $8.64250725378 \mathrm{e}-3$ & $1.75171226089 \mathrm{e}-5$ & $-3.95591220089 \mathrm{e}-4$ \\
$p_{5}$ & $4.90572618639 \mathrm{e}-6$ & $2.85339890535 \mathrm{e}-6$ & $-2.26333221728 \mathrm{e}-4$ & $-4.52001253709 \mathrm{e}-7$ & $1.0423101576 \mathrm{e}-5$ \\
$p_{6}$ & $-9.20435802936 \mathrm{e}-8$ & $-4.21220238424 \mathrm{e}-8$ & $3.3708208075 \mathrm{e}-6$ & $6.58112473668 \mathrm{e}-9$ & $-1.5666898063 \mathrm{e}-7$ \\
$p_{7}$ & $8.68324174519 \mathrm{e}-10$ & $3.56588074378 \mathrm{e}-10$ & $-2.66907742493 \mathrm{e}-8$ & $-5.05435062633 \mathrm{e}-11$ & $1.25731666489 \mathrm{e}-9$ \\
$p_{8}$ & $-3.24809952417 \mathrm{e}-12$ & $-1.28186708997 \mathrm{e}-12$ & $8.73488365216 \mathrm{e}-11$ & $1.59289867849 \mathrm{e}-13$ & $-4.17406548406 \mathrm{e}-12$ \\
\hline
\end{tabular}

Table 4

(b) The polynomial coefficients of cross-section parameters:

$p(x)=p_{n} x^{n}+p_{n-1} x^{n-1}+\ldots+p_{1} x+p_{0}$

\begin{tabular}{lrrrrr}
\hline$p_{i}$ & $\xi_{T}(\mathrm{~cm})$ & $\eta_{T}(\mathrm{~cm})$ & $J_{G}\left(\mathrm{~cm}^{6}\right)$ & $J_{G \xi}\left(\mathrm{cm}^{5}\right)$ & $J_{G \eta}\left(\mathrm{cm}^{5}\right)$ \\
\hline$p_{0}$ & -0.0747046930846 & 1.8650004662 & 19750 & -623 & -163.711581197 \\
$p_{1}$ & -0.0112759392953 & -0.0351671805283 & -1677.41999801 & 33.0994038481 & 4.21781567739 \\
$p_{2}$ & $6.56556746238 \mathrm{e}-3$ & $2.19552862184 \mathrm{e}-3$ & 124.116450758 & -0.488247192948 & -2.92564123694 \\
$p_{3}$ & $-4.24499521168 \mathrm{e}-4$ & $-1.19277692318 \mathrm{e}-4$ & -8.472563615 & $-7.09696129523 \mathrm{e}-3$ & 0.253062515803 \\
$p_{4}$ & $1.17427208476 \mathrm{e}-5$ & $2.90642490327 \mathrm{e}-6$ & 0.379439560195 & $3.7102656835 \mathrm{e}-4$ & $-9.10573923243 \mathrm{e}-3$ \\
$p_{5}$ & $-1.47370081844 \mathrm{e}-7$ & $-3.43373617368 \mathrm{e}-8$ & -0.0100842042704 & $-7.29346816732 \mathrm{e}-6$ & $1.65621883688 \mathrm{e}-4$ \\
$p_{6}$ & $6.85948405639 \mathrm{e}-10$ & $1.5984413365 \mathrm{e}-10$ & $1.54319554418 \mathrm{e}-4$ & $1.04572777032 \mathrm{e}-7$ & $-1.50816797168 \mathrm{e}-6$ \\
$p_{7}$ & & & $-1.25815150944 \mathrm{e}-6$ & $-1.00134130832 \mathrm{e}-9$ & $5.47198751301 \mathrm{e}-9$ \\
$p_{8}$ & & & $4.23271338953 \mathrm{e}-9$ & $4.20180192964 \mathrm{e}-12$ & \\
\hline
\end{tabular}



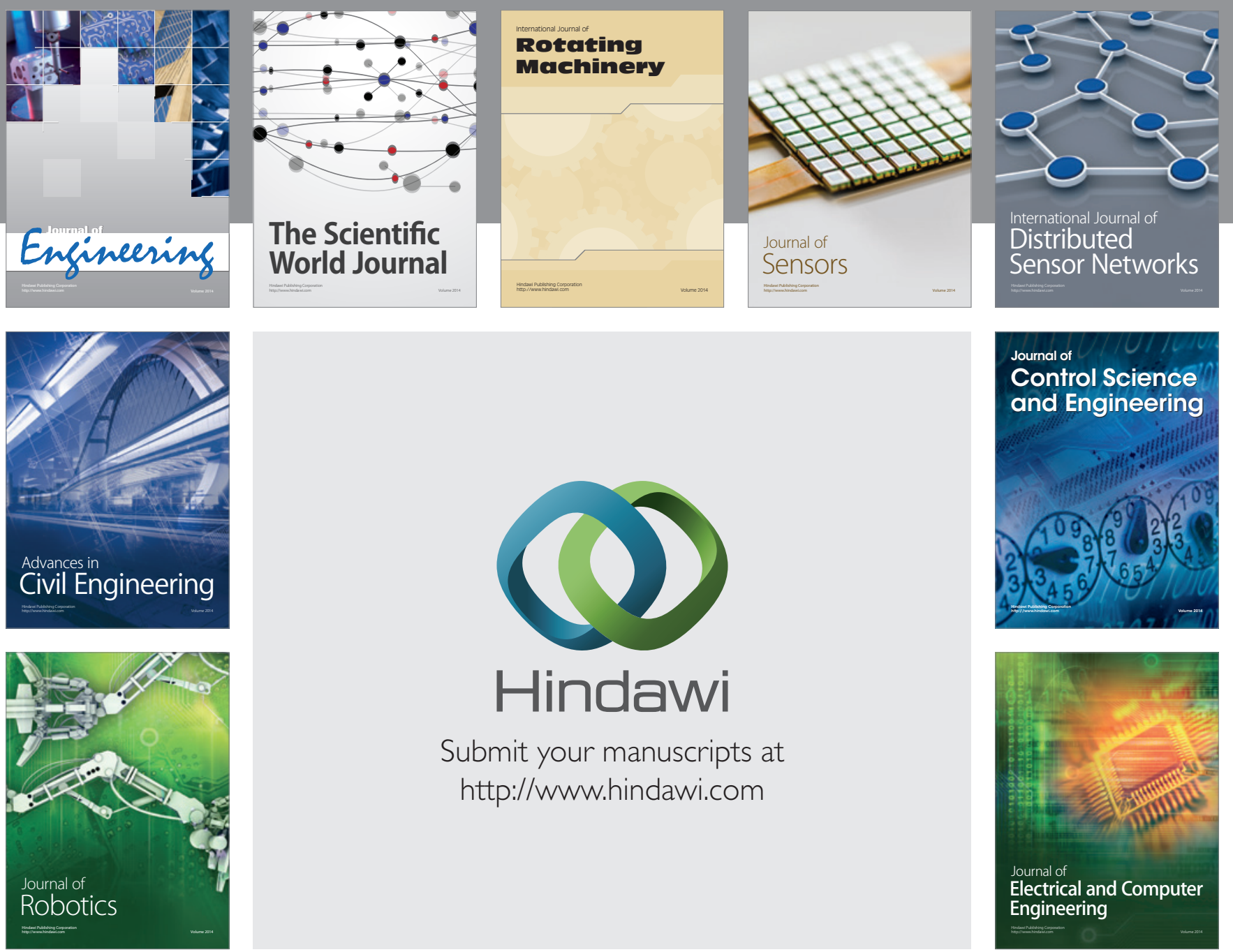

Submit your manuscripts at

http://www.hindawi.com
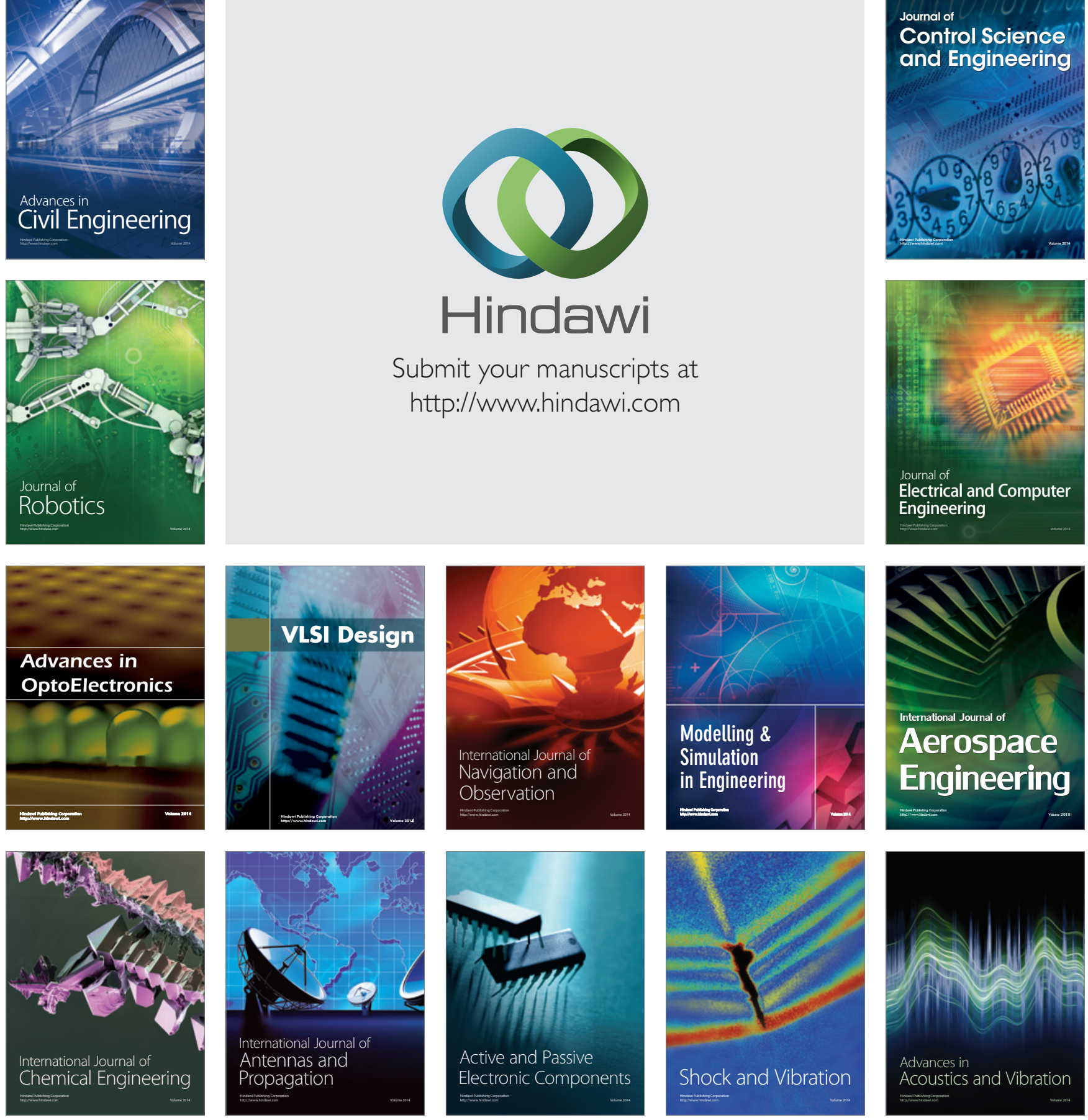